\title{
Alleviating Damage from Epirubicin-Induced Cardiotoxicity with an Increased Dosage of Cardioprotective Magnesium
}

\author{
G. Shukla ${ }^{1}$, A. Waldron ${ }^{1}$, and C. Queenan ${ }^{1}$ \\ 1. Bergen County Academies, Nano-Structural Imaging Lab, 200 Hackensack Avenue, \\ Hackensack, NJ
}

Chemotherapy has been a very crucial treatment method for cancer since its inception. However, its detrimental side effects to other parts of the body have limited its use and are frequently encountered in various stages of the therapy. Epirubicin, classified as an anthracycline antibiotic, is an established chemotherapeutic agent that is known to have detrimental, and at times fatal, effects on the heart [1]. Epirubicin targets and entangles specific DNA sequences of cancer cells to inhibit replication, stopping the unrestricted growth of cancer cells [2]. Cardiomyocytes contain a large number of mitochondria, because of the high demand for energy. Therefore, when the mitochondrial electron transport chain is disturbed by the free radical formation stimulated by epirubicin, apoptosis of cardiac myocytes is induced [3].

In this study, it was hypothesized that magnesium would relieve the cardiotoxic effect of epirubicin. Magnesium is frequently released into the extracellular matrix to repair damaged cells. It has been suggested that the cardioprotective effects of $\mathrm{Mg}^{2+}$ may be due to antagonization of $\mathrm{Ca}^{2+}$, which inhibits $\mathrm{Ca}^{2+}$ influx and $\mathrm{Ca}^{2+}$ overload. However, during ischemia, the intracellular $\mathrm{Mg}$ concentration decreases. This suggests that high extracellular $\mathrm{Mg}^{2+}$ concentrations prevent $\mathrm{Mg}^{2+}$ efflux from the intracellular space [4].

MDA-MB-231 human breast cancer cells were used to gauge the effectiveness of epirubicin in vitro. The five concentrations of magnesium chloride used ranged from 1 $\mu \mathrm{M}$ to $10 \mathrm{mM}$ by factors of 10 . The concentrations of epirubicin ranged from $200 \mathrm{nM}$ to $2 \mathrm{mM}$ by factors of 10 . Cell viability was measured after magnesium and epirubicin treatment using CellTiter 96® Proliferation Assay, which uses the percent of relative absorbance to determine the amount of viable cells per condition. Scanning electron microscopy was used to analyze cell death and proliferation, along with any morphological changes that occurred due to treatments.

The results from experimentation indicated that epirubicin decreased the viability of breast cancer cells by up to $44 \%$ of the control. This indicates the efficacy of epirubicin as a chemotherapeutic drug. However, the magnesium solutions had little to no effect on the proliferation of the MDA-MB-231 breast cancer cells, which was expected. These tests were proven statistically significant and the results showed little to no variability. Further tests must be performed with cardiomyocytes in order to solidify the role of magnesium as a cardioprotective agent. Overall, magnesium shows promise as an effective agent for cancer therapy while protecting the heart from epirubicin-induced injury. 


\section{References}

[1] M. Ryberg, et al., Journal of the National Cancer Institute 100 (2008), 1058-1067.

[2] R. Olinski, et al., Molecular Pharmacology 52 (1997), 882-885.

[3] N. G. Fisher, et al., Postgraduate Medical Journal 75 (1999), 265-268.

[4] M. Watanabe, et al., Journal of Clinical \& Experimental Cardiology 9 (2004), 181.

[5] J. Kirkels, et al., Journal of Molecular \& Cellular Cardiology 21 (1990), 1209-1218.

[6] P. Spallarossa, et al., Journal of Pharmacology \& Exp. Therapeutics 332 (2010), 87.

[7] The authors would like to acknowledge the administration of the Bergen County

Technical School District for their continued support of the research program.

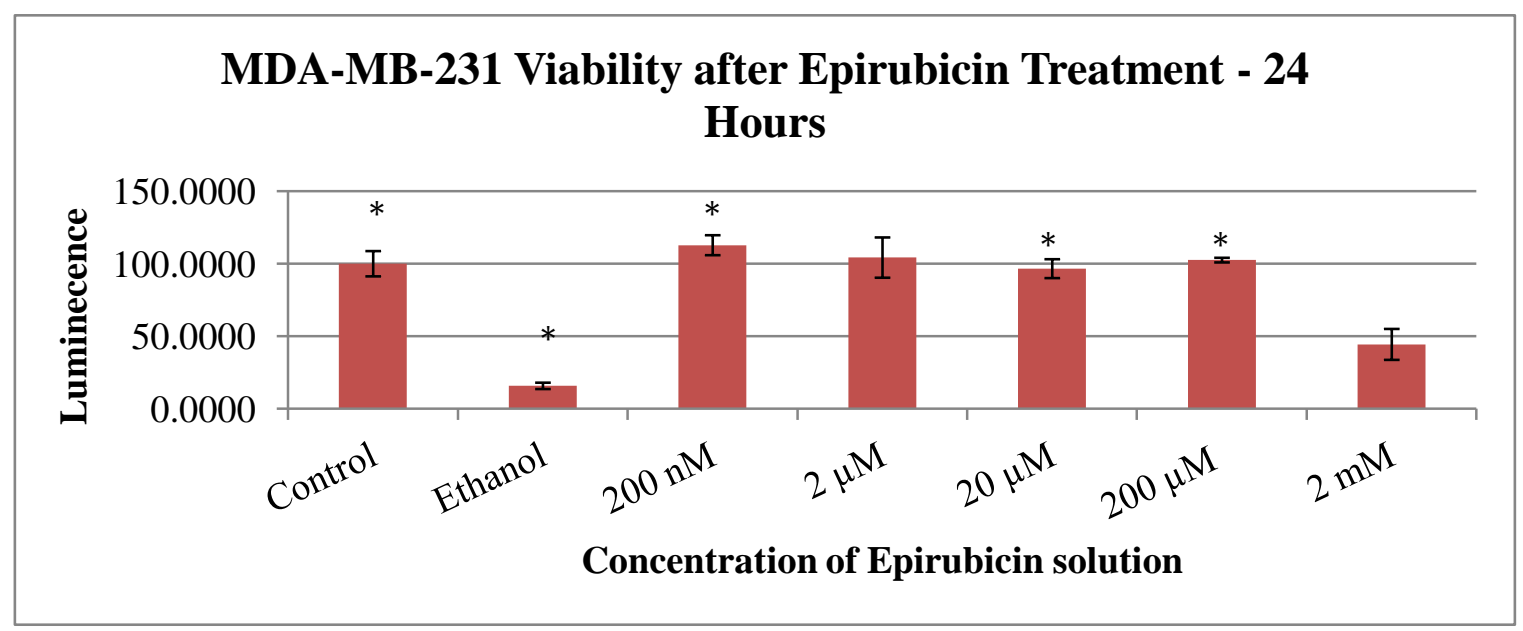

Figure 1: MDA-MB-231 viability after epirubicin treatment for 24 hours. Data is represented by mean $\pm \mathrm{SD}(\mathrm{n}=3)$. $*=\mathrm{p}<0.005$.

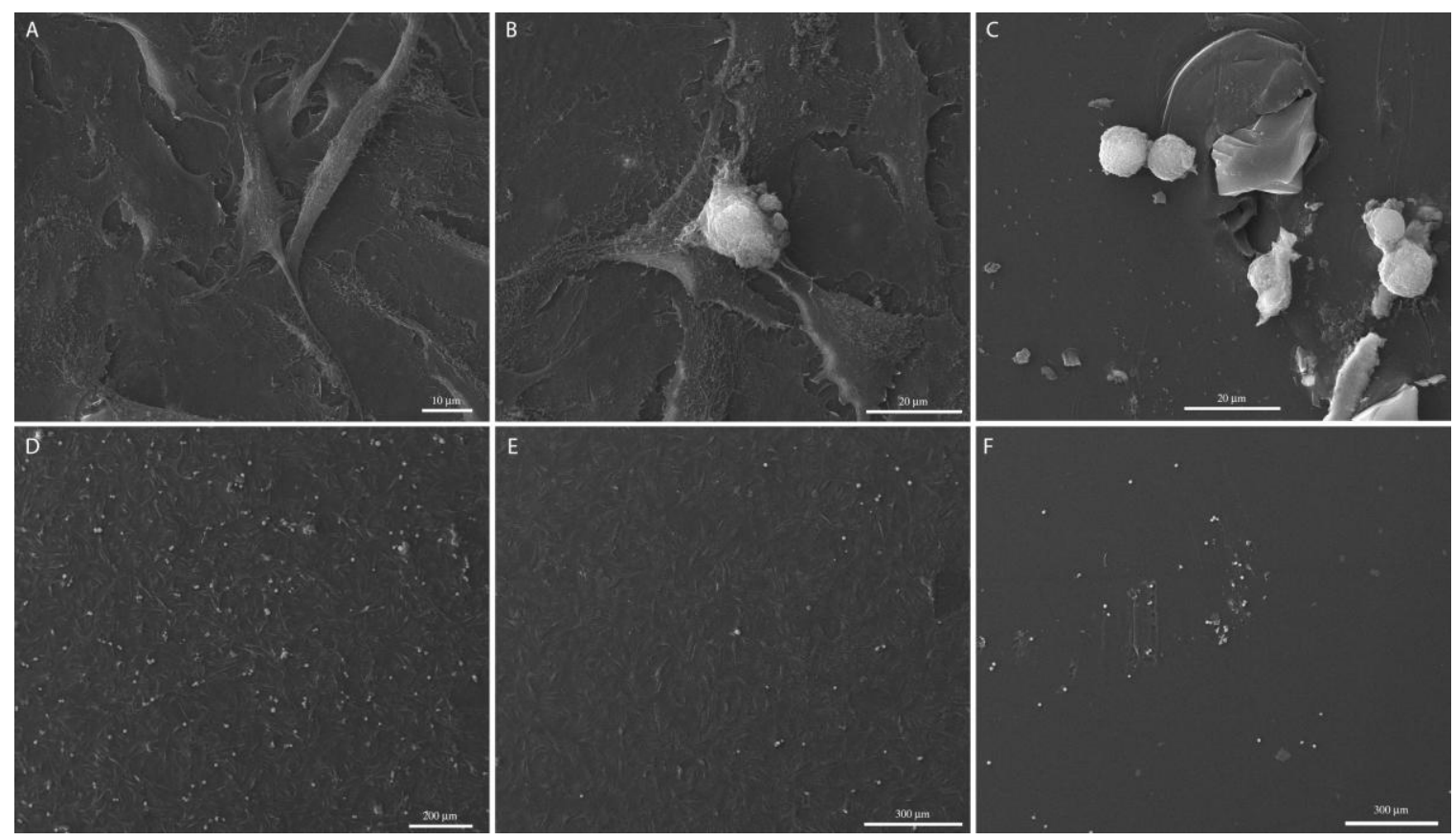

Figure 2: SEM images of MDA-MB-231 cells treated with epirubicin. Images A, D: 200 $\mathrm{nM}$ epirubicin. Images B, E: $20 \mu \mathrm{M}$ epirbucin. Images $\mathrm{C}, \mathrm{F}: 2 \mathrm{mM}$ epirubicin. 